\title{
Modelagem de Regras de Negócios Integrada ao Projeto de Software Orientado a Processos de Negócios
}

\author{
Cristine do C. S. Bueno de Moraes, Luiz Camolesi Júnior \\ Faculdade de Ciências Exatas e da Natureza (FACEN) \\ Universidade Metodista de Piracicaba (UNIMEP) \\ Piracicaba - SP - Brasil \\ cristine.moraes@uol.com.br, 1camoles@unimep.br
}

\begin{abstract}
The relevance of methods that allow to extract the user's knowledge is in the complexity of the systems development with the systemic recognition of the users' knowledge, as well as providing its distribution or modernization in the environments, consisting of the integration and attendance to the requirements, technology demand of the information and corporate administration of business. This work presents a solution for the integration of the business rules modeling in a methodology of software development based on business processes, with emphasis in the need to identify and to already readapt methods existent. The result is a methodology that aids this process, focusing the business administration and the warranty of the competitive advantage for the organizations.
\end{abstract}

Resumo. A relevância de métodos que permitam extrair o conhecimento do usuário reside na complexidade do desenvolvimento de sistemas com o reconhecimento sistêmico do conhecimento dos usuários, bem como de proporcionar sua distribuição e atualização no ambiente, consistindo na integração e atendimento aos requisitos, demanda tecnologia da informação e gestão corporativa de negócios. Este trabalho apresenta uma solução para a integração da modelagem de regras de negócios em uma metodologia de desenvolvimento de software baseada em processos de negócios, com ênfase na necessidade de identificar e readaptar métodos já existentes. O resultado é uma metodologia que auxilie neste processo, focando a gestão de negócios e a garantia da vantagem competitiva para as organizações.

\section{Introdução}

O contexto atual que envolve empresas e organizações caracteriza-se por ser um ambiente dinâmico e complexo, que tem como fundamento principal a eficácia operacional, a disseminação e controle sobre a estratégia de seus processos de negócios. Tal fato implica no uso cada vez mais freqüente da Tecnologia da Informação - TI como um tipo de recurso estratégico para o suporte eficaz e eficiente na tomada de decisões. No entanto, o desenvolvimento e/ou composição de tais recursos envolve um aspecto amplo, onde a definição de parâmetros, processos e características específicas a cada ambiente modelado demanda um alinhamento estreito entre TI e a área de negócios (Abu-Hanna \& Jansweijer, 1994; Kelly et al, 1999; Marshall, 2000). 
Desta constatação é possível uma especialização dos fatos que revela que entre os desafios no desenvolvimento de Sistemas de Informação - SI, parte integrante das soluções em Tecnologia da Informação e co-responsável pelas respostas ao dinamismo das áreas aplicadas, está a metodologia que orienta as etapas do desenvolvimento dos Sistemas de Informação, particularmente nas atividades relacionadas à:

- Representação dos processos de negócios;

- Visão da estratégia da organização, sintetizada através das regras e política de negócios e que freqüentemente não se encontram representadas no processo empresarial (Ross, 2000);

- Identificação e captura dos conhecimentos informais aplicados na condução das estratégias de negócios.

Decorrente do exposto verifica-se a relevância do aperfeiçoamento contínuo das metodologias existentes para que o desenvolvimento e especificação de Sistemas de Informação contemplem o compartilhamento e gestão do conhecimento em ambientes de negócios, através da integração e consistência de dados, processos e conhecimento de negócios em uma visão unificada da empresa em todas as suas extensões (Kelly et. al, 1999; Marshall, 2000).

\section{Regras de Negócios}

Em quaisquer organizações ou instituições pode ser verificada a existência de dimensões-chaves (elementos estratégicos) que compõem a dinâmica das atividades realizadas pelas pessoas, dentro de seus contextos culturais, filosóficos, estruturais e etc. Em todos estes contextos, as Regras de Negócios estão presentes como uma dimensãochave dedicada ao direcionamento e regulamentação de ações em uma organização.

Regras de Negócios são expressões envolvendo pessoas, dados (objetos) e processos de trabalho de uma organização (Ross, 2000) e definidas explicitamente para orientar as interações entre pessoas e pessoas e objetos, segundo definições dos documentos que estabelecem os objetivos, metas e missão organizacional da organização. Desta forma, a motivação para a elicitação das Regras de Negócios está na sua implantação em Sistemas de Informação para auxiliar no suporte e manutenção da consistência das ações destes sistemas, assegurando a integridade e o suporte à interação entre os usuários e permitindo atingir as necessidades da organização (Anderson et al, 1997).

A importância da modelagem das Regras de Negócios em uma metodologia de desenvolvimento de software reside na captura e identificação de normas estabelecidas dentro de uma ordem de escalonamento determinada, visando desse modo servir de suporte no processo de gestão estabelecido pela organização (Sauer \& Bruns, 1997).

No entanto, a dimensão das regras para organizações de negócios constitui um contexto dinâmico e mutável, devendo ser adaptada em decorrência de necessidades identificadas através de variáveis externas e decisões internas gerenciais (Hwang, 2003; Ross, 2004; Kelly et al, 1999; Marshall, 2000). Isto implica na compreensão do contexto do ambiente, visando a integração de sua perspectiva de evolução como uma etapa decisiva em projetos de software para organizações de negócios. 


\section{Trabalhos Relacionados}

A modelagem de regras de negócios tem sido objeto de estudo por diferentes pesquisadores em suas linhas de pesquisa, visto a complexidade da compreensão e necessidade de alinhamento entre os aspectos da organização de negócios e a área de TI (Tecnologia de Informação). Entre estas pesquisas encontram-se os trabalhos realizados por Abu-Hanna \& Jansweijer (1994), Kelly et al (1999), Marshall (2000), Ross (2000), Hwang (2003), Ross (2004), McGovern (2002) que abordam a importância da análise e captura do contexto empresarial para o desenvolvimento de projetos de software particularmente envolvendo a modelagem e implementação de Regras de Negócios. No entanto, nestas pesquisas a elicitação e integração das regras aos demais componentes de um SI não está disseminada pelas diferentes etapas do projeto.

Reconhecendo ser esta uma orientação fundamental para a qualidade de um SI, este trabalho aborda duas metodologias consagradas (resumidamente apresentadas abaixo) buscando integra-las na proposição de uma metodologia com os alinhamentos citados e a valorização da modelagem de regras de negócios.

- Processo de Modelagem empresarial : a modelagem empresarial definida por Marshall (Marshall, 2000) é uma das poucas obras que mencionam a importância da engenharia de negócios frente a aspectos tecnológicos do projeto. O objetivo principal de Marshall é expor a importância da captura de informações para o negócio sobre o qual a organização está estruturada, através da identificação dos fluxos principais de valores, informações e relacionamentos entre diferentes entidades e, conseqüentemente, obtendo informações sobre políticas e diretrizes que implicam em regras e processos de negócios inerentes a cada organização.

- Processo Unificado de Desenvolvimento de Software (USDP) (Jacobson, Booch e Rumbaugh, 1999): processo de desenvolvimento de software que tem por objetivo transformar os requisitos dos usuários em um sistema de software, através de um conjunto de atividades iterativas e inter-relacionadas. O objetivo principal consiste no auxílio aos desenvolvedores no projeto e desenvolvimento de um sistema que satisfaça as necessidades dos usuários. Apoiado na Unified Modeling Language - UML (Booch, Rumbaugh, Jacobson, 2000), esta metodologia é utilizada para permitir flexibilidade e extensibilidade ao projeto, decorrente de suas características que permitem uma grande variedade de estratégias de ciclo de vida do projeto, entre as quais a seleção de artefatos que devem ser produzidos, a definição de atividades e atores (papéis).

\section{Metodologia}

A metodologia proposta possui etapas, fases, atividades, procedimentos e documentos que são integrados entre si. No contexto deste trabalho de pesquisa, definiu-se a importância da utilização de uma metodologia apoiado no paradigma da orientação a objetos incorporando a modelagem de negócios. Na definição desta metodologia, a proposta de Marshall foi utilizada para o desenvolvimento do modelo de negócios, tendo importância na definição dos elementos e política. A USDP foi utilizada como metodologia "guia" para a proposta, abordando todas as etapas do projeto; e a UML 
utilizada como linguagem para modelagem de software. A metodologia é dividida em 4 etapas e respectivas sub-etapas representadas na Figura 1.

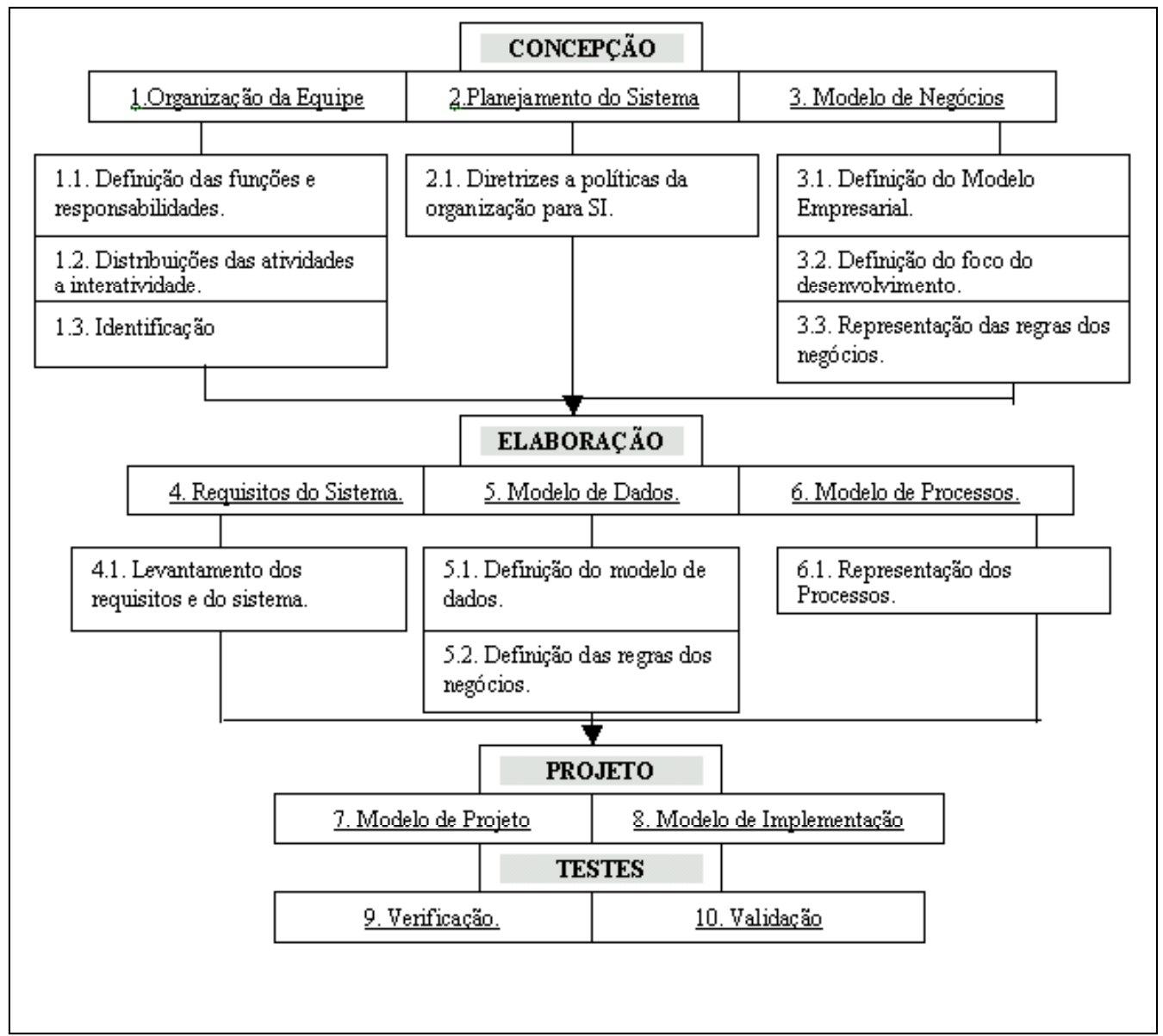

Figura 1. Visão geral da metodologia.

\subsection{Concepção}

Esta etapa constitui na idealização e planejamento do sistema onde é o definido o escopo sobre o qual o software será desenvolvido. Dividida em Organização da Equipe, Planejamento do Sistema e Modelo de Negócios, a importância desta etapa reside na compreensão do contexto da organização pela equipe do projeto, sendo fundamental para a compreensão das regras de negócios bem como seu relacionamento nos processos inerentes a cada organização em particular. Na fase de Organização da Equipe são consideradas as habilidades e disponibilidades de envolvimento de cada ator com o projeto, definindo-se uma equipe com estrutura de organização heterogênea. $O$ Planejamento do Sistema envolve a estratégia da empresa, no qual é realizado o levantamento e os estudos específicos que possibilitam a tomada de ações de acordo com as necessidades e objetivos da empresa.

A fase do Modelo de Negócios adotada a abordagem proposta por Marshall (2000), incluindo documentos que serviram de base para o projeto. São eles: a) definição da estrutura de rede de valor e informação; b) descritivo de subsistemas e 
macro-atividades; c) identificação do subsistema; d) representação de regras de negócios. Importante destacar aqui a importância do item d, visto que neste momento são identificados de forma macroscópica os relacionamentos entre as diferentes áreas da organização, as regras de negócios aplicáveis a cada área, bem como a ordem seqüencial de escalonamento e os sistemas legados existentes sobre as mesmas, através da geração de dois documentos: descritivo de parâmetros e regras de negócios utilizados e o mapa de processos.

\subsection{Elaboração}

Nesta fase ocorre a execução do planejamento ou elaboração do sistema, através da especificação de suas características. Esta fase é subdividida em: Requisitos do Sistema; Modelo de Dados e Modelo de Processos. O levantamento dos requisitos, realizado na fase de Requisitos do Sistema, deve ser considerado essencial para a consistência do projeto, por envolver o comportamento dos processos do sistema.

O Modelo de Dados tem seu foco na definição de um modelo de dados para armazenamento e consistência das informações que compõem um banco de dados do qual se extraem informações para a tomada de decisões de negócios, independentemente do seu grau de importância (operacional ou estratégica). Neste contexto é importante ressaltar que a gestão de informação e a modelagem dos componentes do projeto tecnológico devem envolver o fluxo do processo de negócios, onde modelagem de processos, modelagem de conhecimento e modelagem de dados necessitam ser desenvolvidas de forma integradas pela sua característica de interdependência.

A importância da etapa de elaboração no Modelo de Processos destaca-se por envolver a identificação da regras e processos que são interdependentes e possuem sua associação e acionamento determinado por variáveis externas. Tais variáveis são recebidas por diferentes stakeholders (colaboratores) envolvidos no processo, que realizam inferências nos dados, agindo sobre o processo, a partir de requisitos estabelecidos pela organização.

\subsection{Projeto}

Nesta etapa abordam-se as várias visões da arquitetura, através da utilização dos diagramas da UML confeccionados durante a etapa de elaboração. Esta etapa é subdividida em: Modelo de Projeto e Modelo de Implementação. Apesar de sua importância na implementação da metodologia proposta como um todo, no foco deste trabalho torna-se relevante apenas esclarecer que é nesta etapa que ocorre a transferência do domínio analisado (regras processos e dados) para a construção e implementação de uma solução de TI para a organização.

\subsection{Testes}

Nesta etapa é executada a fase de implantação do produto ao domínio, incluindo a Verificação e Validação do sistema. Nesta etapa é realizado o refinamento do projeto pela equipe de stakeholders envolvidas com o desenvolvimento. As decisões de consolidação exigem da equipe o refinamento para reconhecimento dos processos em suas diferentes inferências, sendo possível adquirir uma melhor visão sobre as necessidades reais da organização. 
Durante cada fase são especificados documentos que funcionam como suporte para as fases seguintes que serão executadas. Como pode ser verificado no modelo proposto, o refinamento do projeto ocorre nas diferentes etapas, sendo que deve ser dado um destaque importante à $1^{\mathrm{a}}$ e $4^{\mathrm{a}}$ etapa, onde são identificados os relacionamentos entre regras e processos de negócios no domínio analisado.

\section{Estudo de Caso: O sistema SURREAL}

O estudo de caso realizado em uma indústria de médio porte do setor químico permitiu experimentar os problemas inerentes a especificação de um projeto de sistema para as organizações empresariais onde processos e regras estão inclusas em declarações explícitas e implícitas (Ross, 2000), sendo fundamental a sua correta identificação para que o produto atenda as necessidades das organizações (Mcgovern, 2002).

A metodologia proposta foi aplicada em um Sistema de Informações Corporativas - SIC denominado SURREAL, que vincula os processos empresariais entre os diferentes setores da organização empresarial. Particularmente, a experimentação da metodologia foi realizada no sub-sistema de gestão de clientes que possui diversos empregos na empresa, desde o suporte ao contato informal até o acompanhamento da evolução dos parâmetros comerciais de um cliente. Esta evolução é definida através de indicadores e parâmetros que especificam o grau de relacionamento de cada cliente com a indústria. Estes parâmetros possuem uma classificação especifica para a organização, embora englobem sempre o mesmo conceito. Nas organizações de um modo geral, mensurar, analisar e avaliar são diferentes fases do processo de relacionamento com o cliente, desenvolvendo e fortalecendo estratégias para o aumento de negócios.

No estudo de caso analisado, parâmetros foram definidos através de diferentes indicadores, entre os quais destacam-se:

(1) O ranking de faturamento escalonado através de níveis,

(2) O potencial de faturamento, escalonado através de um ranking;

(3) A classificação por comportamento de compra;

(4) A classificação por estratégia de atuação;

(5) A classificação por segmento;

(6) O status (grau de relacionamento existente com a organização).

Estes indicadores possibilitam análises diferentes que dão suporte a decisão em diferentes setores conectados. Tais indicadores também permitem uma visão unificada dos processos, visto que, por tratar-se de uma empresa que atua no setor B2B (business to business), no processo de relacionamento com o cliente são envolvidos diferentes stakeholders por ambas as partes, o que gera uma rica e complexa rede de relacionamentos.

Uma das regras que definem a forma e o responsável pelo atendimento do cliente é o grau de relacionamento com o mesmo. Uma empresa com um relacionamento de negócios não ativo, ou seja, sem a efetivação de vendas é atendida através de um processo denominado prospecção, onde estão envolvidos diferentes stakeholders e regras que são determinadas através dos indicadores 4,5 e 6 . No momento em que o relacionamento torna-se mais intenso, é possível identificar-se o parâmetro 2 e 3 e pode ocorrer a alteração de stakeholders envolvidos e de regras de negócios. A partir da efetivação do processo de compra, com os módulos envolvidos com o módulo analisado 
(módulo faturamento e módulo cobrança), o mesmo cliente tem seu status alterado e ocorre uma evolução do relacionamento, denominado administração de vendas.

Neste novo processo existem outras regras de negócios e stakeholders envolvidos, que são determinados pelos indicadores (1), (2), (3), (4), (5) e (6), sendo que os mesmos podem ser alterados com a evolução. Assim, são variáveis externas relacionadas com cada cliente ou empresa em particular e a evolução das mesmas, sintetizadas através dos indicadores mencionados, que determinam os processos e regras de negócios envolvidos no módulo Dossiê de Clientes.

O sistema SURREAL suporta uma interação dinâmica e direcionada. Isto porque, são os stakeholders indicados pelas próprias regras inclusas no sistema e no processo modelado que alimentam o mesmo, possibilitando sua dinâmica e acúmulo de conhecimento. A tabela 1 mostra o acúmulo do conhecimento possibilitado através do produto desenvolvido a partir do modelo proposto, indicando as inferências que ocorrem a partir das variáveis externas. A partir das informações originadas das variáveis externas, foram gerados ou atualizados os indicadores, acionando regras e processos vinculados que possibilitam novos indicadores ou sua atualização. Isso ocorre de forma dinâmica, visto que os indicadores demonstrados também possibilitam ações originadas a partir dos stakeholders, podendo gerar ações junto as variáveis externas e ocasionar uma nova posição e atuação da mesma no contexto.

Tabela 1. Demonstrativo do conhecimento acumulado.

\begin{tabular}{|c|c|c|}
\hline Variável Externa & Indicadores Gerados & Inferência em \\
\hline Cliente Não Ativo & (4) (5) (6) & $\begin{array}{l}\text { Stakeholders } \\
\text { Processos }\end{array}$ \\
\hline $\begin{array}{l}\text { Cliente Não Ativo } \\
\text { (em trabalho) }\end{array}$ & $\begin{array}{lllll}(2) & \text { (3) } & \text { (4) } & \text { (5) } & \text { (6) }\end{array}$ & $\begin{array}{l}\text { Indicadores } \\
\text { Stakeholders } \\
\text { Processos } \\
\text { Cliente (Variável Externa) }\end{array}$ \\
\hline Cliente Ativo & $\begin{array}{llllll}(1) & (2) & (3) & \text { (4) } & \text { (5) } & \text { (6) }\end{array}$ & $\begin{array}{l}\text { Indicadores } \\
\text { Stakeholders } \\
\text { Processos } \\
\text { Cliente (Variável Externa ) }\end{array}$ \\
\hline
\end{tabular}

\section{Considerações Finais}

Este trabalho é resultado de um estudo sobre as implicações de processos e modelos de dados e negócios em projetos de desenvolvimento do sistema, visando a definição de uma metodologia para especificação.

Com a aplicação do estudo foi possível verificar a viabilidade da aplicação da metodologia na relação direta entre os objetivos teóricos e científicos e o proveito por organizações, neste caso, a utilização em sistemas de suporte a processos empresarias e de negócios. A proposição da metodologia abrangeu os seguintes objetivos e resultados:

- Análise das principais metodologias e modelos existentes para processos de planejamento, especificação de projetos de Sistema de Informação Corporativos, baseados na modelagem e domínio do conhecimento;

- Melhoria no atendimento aos requisitos dos usuários e da otimização de recursos, com o reconhecimento, identificação e estruturação do funcionamento 
do modelo empresarial, proporcionado através de captura e modelagem das regras e processos de negócios estabelecidos em uma ordem de escalonamento pré-determinado;

- Identificação dos requisitos necessários ao desenvolvimento de um modelo que proporcione a integração de dados e processos de negócios para as organizações na especificação de produtos de software.

Com o emprego da USDP na proposição da metodologia deste trabalho, pode-se notar que apesar do detalhamento considerável no processo, não está formalizada a redundância de informações nos diversos modelos de UML Isto acarreta dificuldades adicionais na compreensão dos modelos por profissionais especialistas em processos de negócios (stakeholders-chaves), prejudicando a inferência e auxílio dos mesmos no processo de captura e modelagem do conhecimento. Apesar desta análise não estar relacionada diretamente com a proposta deste trabalho, a deficiência relatada tornar-se-á uma linha de estudo futura dos autores.

\section{Referências Bibliográficas}

Abu-hanna, A; Jansweijer, W. (1994) "Modeling Domain Knowledge Using Explicit Conceptualization”. IEEE Expert Intelligent Systems, V. 9 , N. 5., p. 53-64.

Anderson, E., Bradley, M.; Brinkro R. (1997) "Use case and business rules: styles of documenting business rules in use cases”. ACM Press, USA, p. 85-87

Booch, G; Rumbaugh, J; Jacobson, I (2000) The Unified Modeling Language User Guide. Addison-Wesley.

Jacobson, I; Booch, G; Rumbaugh, J; (1999) The Unified Software Development Process. Addison -Wesley.

Hwang, J.D. (2002) Information Resources Management: New era, New Rules. IT Professional. V. 4, N. 6, p 9-17.

Kelly, S.; et al. (1999) "Focus Issue on Legacy Information Systems and Business Process Change: A Business Perspective of Legacy Information Systems". Communications of the Association for Information Systems. V. 2.

Marshall, C. (2000) Enterprise Modeling With UML - Designing successful software through business analysis. Addison Wesley Longman.

Matheus, M et al. (1993) "Systems for Knowledge Discovery in Databases". IEEE Transactions On Knowledge And Data Engineering, V. 5, N. 6, p. 903-913.

Mcgovern, F. (2002) Managing Software Projects with Business-Based Requirements. IT Professional. V. 4, N. 5, p 18-23.

Ross, R. G. (2000) "Expressing Business Rules". ACM SIGMOD International Conference on Management of Data, pp. 515-516.

Ross, R. G. (2004) "The Notion of the Perfect Platform-And Whot it Means for System Models". Business Rules Journal, V. 5, N. 5.

Sauer, J; Bruns, R. (1997) "Knowledge-Based Scheduling Systems in Industry and Medicine”. IEEE Intelligent Systems, V. 12, N. 1, p. 24 -31. 